\title{
An Induced Fluorescence Detecting System with Autofocus Electrically Tunable Len
}

\author{
Chang Zhou, Xiaoming $\mathrm{Hu}$, and Ya Zhou
}

\begin{abstract}
Confocal microscopy is a method for induced fluorescence detecting system which is widely used in biochip and microfluidic chip. However, the sensitivity is influenced seriously by the installation uncertainty which leads to the detecting plane being away from the object plane. A new autofocus confocal system with an electrically tunable liquid lens is designed to adjust the detecting plane automatically through changing the focal length of the object electrically tunable lens group until light focus on the center of detection channel by a camera. This design makes it possible to improve the accuracy of confocal microscopy in a small space.
\end{abstract}

Index Terms-Confocal microscopy, electrically tunable liquid lens, automatic focusing.

\section{INTRODUCTION}

Confocal microscopy is a method which is usually used in induced fluorescence detection. It can reduce spurious signal interference with a hole at the focal point which can significantly improve the sensitivity of the detector. However due to processing limitations, the actual size of the mirofluidic chip is variable to a certain extent in the fluorescence information collection, which affects the detection sensitivity.

In order to solve this problem, many schemes have been proposed which can be sorted into following kinds. In one kind, images are stored in computer. They can be observed after complex enhancement algorithm [1]-[3]. In another kind, a precision displacement system is used to move the lens group to adjust the focusing surface of the confocal system [4], [5]. What's more, an array of remote focusing systems was shown to allow multi-layer imaging [6], [7]. This system uses the sequential timing of laser pulses that can be focused at different focal depths and assigns the detected fluorescence signal to the correct plane of origin accordingly. But all kinds of schemes must rise to a challenge that they can hardly be integrated in a small system, leading to an undesirable size of the confocal system.

Such approach exhibits limitations concerning speed, reliability and costs. Instead of using complex mechanics, the z-position of a laser spot can be controlled by using an electrically focus tunable lens, and such system can be made more compact, reliable and less expensive.

A new confocal system with an electrically tunable lens is

Manuscript received January 9, 2015; revised March 10, 2015. This work is supported by the Beijing Higher Education Young Elite Teacher Project (YETP1170).

The authors are with the School of Optoelectronics, Beijing Institute of Technology, Beijing, 100081, China (e-mail: 158494592@qq.com, bithxm@bit.edu.cn, zhouya@bit.edu.cn). proposed in the paper. It can adjust its focal length of the liquid lens according to the collected image edge information. In this way, we can focus light on detection channel in the center of the microfluidic chip. This system can improve confocal microscopy in a small space. How to increase the automatic focusing precision becomes our primary concern. There are two main influencing factors in the processing of automatic focusing, accuracy of sharpness evaluation and the stability of hardware. In this paper, these two parameters should be improved to optimize confocal microscopy.

\section{SYSTEM PRINCIPLE}

\section{A. The Principle of Confocal System}

In a conventional optical microscope, the observed object plane is illuminated by field source, so the imaging quality of the observed object will be influenced by the adjacent points. In laser scanning confocal microscope, every point on the focal plane is scanned with a laser beam irradiating on pinhole to form a point light source. The irradiated points on the specimen image at the detecting pinhole through confocal optical system, and the image can be received by an image sensor such as CCD or CMOS. As the illuminating pinhole and the detecting pinhole are conjugate relative to the focal plane of object lens, the points on the focal plane will focus on both pinholes at the same time. Meanwhile the points off the focal plane can't image at the detection pinhole, so an optical cross section is collected with confocal microscope. In this way, the imaging quality of specimen can be improved.

The principle of confocal system can be seen from the Fig. 1.

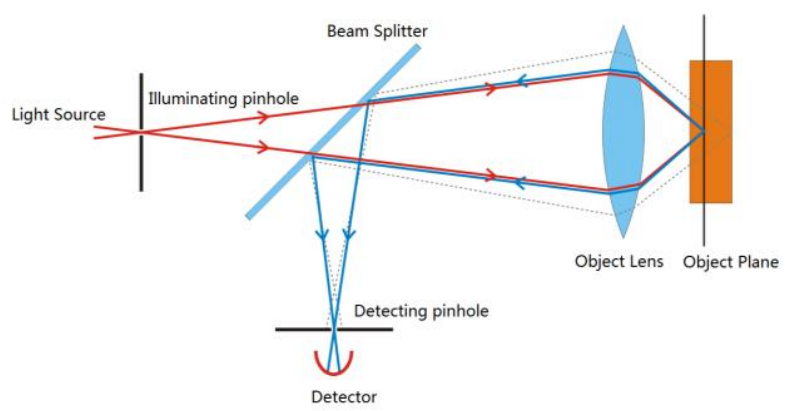

Fig. 1. The principle of confocal microscopy.

In this paper, we replace the object lens in Fig. 2 with a tunable liquid lens which can adjust its focal length according to the definition information collected by a detector [8]. The adjustment won't stop until the detector collects a distinct image. 


\section{B. The principle of Liquid Lens}

The Optotune lens is based on shape-changing, as illustrated in Fig. 2. A container which is filled with an optical fluid and sealed off with an elastic polymer membrane is the main part of the lens. The focus length of the lens is proportional to the pressure in the fluid. It contains an electromagnetic actuator which is used to exert pressure on the container. Hence, the focal distance of the lens is controlled by the current which flows through the coil of the actuator. Thus, the repeatability precision is related with the accuracy of constant current source [9], [10].

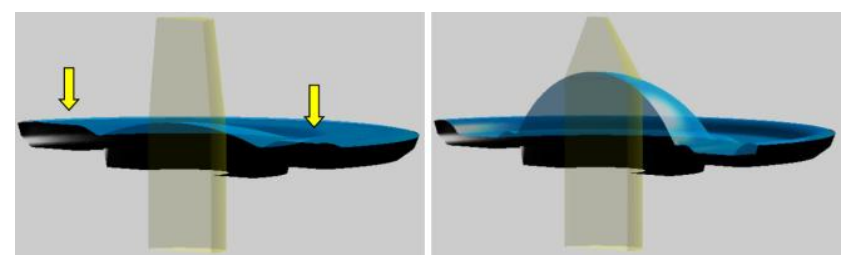

Fig. 2. Principle of liquid lens.

\section{HARDWARE DESIGN}

In order to ensure the stability and accuracy of final driving circuit which is used to control focal length of the Optotune lens, a constant current source is needed with output range from $0 \mathrm{~mA}$ to $300 \mathrm{~mA}$, and power range is 0 to $1.1 \mathrm{~W}$.

To meet these requirements, there are three standard driving methods. The first one is to use the constant current source which is designed for laser diode, such as NT56-804 or NT84-355, and the second approach is to using PWM to control with the frequency of $20 \mathrm{KHz}$ to $50 \mathrm{KHz}$, and the third suggestion is to use the high precision 12-bit current source as the core components such as ADN8810 and design a new driver.

In our implement, an ADN8810 is adopted as the chip for the driver. The ADR292 is a low noise, micropower precision voltage references which supplies a $4.096 \mathrm{~V}$ reference volt for ADN8810. So we choose the third method to drive the liquid lens, and finish the following hardware design which is shown in Fig. 3 and Fig. 4.

\section{SOFTWARE DESIGN}

As the feedback information of the closed-loop system, image sharpness is a key factor which can determine the accurancy of focusing. So how to evaluate image sharpness is discussed in this paper, which can decide the autofocus precision of the system. Because the system is based on DSP, running time should also be considered. The software must take both the accuracy and real-time performance into account.

Before the image processing begins, the images are needed to be denoised. As there is a laser light source, the collected image is a gray image. And most of the noise is white noise caused by the detector, so we use Gauss filter here to remove such noise. In this way, the image can become smoother, and reduce the error of evaluation.

The software design can be divided into two parts. The first part is to find a reasonable standard for evaluating the image sharpness. This standard is used as the system feedback information. So it can determine the accuracy of confocal system. The second part is the auto focusing algorithm. An efficient algorithm can significantly reduce the focusing time, so that the system can achieve the requirement of real-time. And an improved algorithm is proposed according to the character of this Optotune lens.

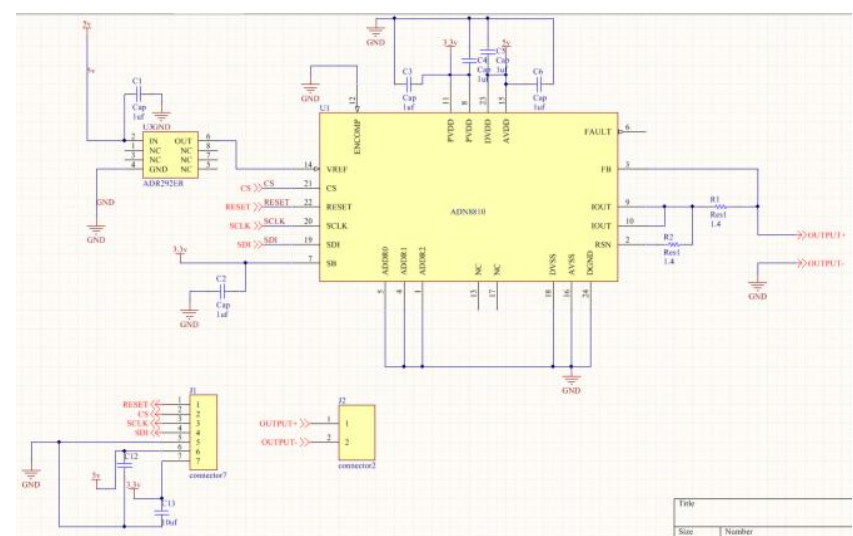

Fig. 3. Schematic diagram of the driver.

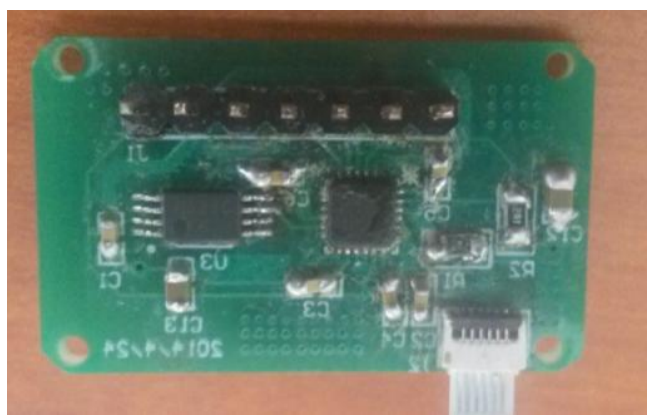

Fig. 4. The driver circuit.

\section{A. Sum of Laplace Transforms}

There are many operators which are able to find edge information of the image. With the operator, different algorithm is proposed to evaluate the image definition. The more edge information does the image has, the higher the definition is. Then we can get a merit function definition with focal length. The function is the reference of autofocus in the following steps.

Auto focusing evaluation function is described as following.

1) Peak gray comparison method. Assume that the image function is not negative $(f(x, y) \geq 0)$, then we can draw the evaluation parameter that $E=\max \left[f\left(x_{i}, y_{i}\right)\right]$. This algorithm regarded the value of E represents the image definition. It's a simple algorithm, but it's only fit for single point imaging

2) Contrast comparison method. This algorithm is used for plane scanning with the evaluation parameter $\mathrm{C}$ which means contrast

$$
\begin{array}{r}
C=\sum_{j=0}^{M} \sum_{i=0}^{N}\left[f\left(x_{i}, y_{j}\right)-f\left(x_{i-1}, y_{j}\right)\right]+ \\
\sum_{j=0}^{M} \sum_{i=0}^{N}\left[f\left(x_{i}, y_{j}\right)-f\left(x_{i}, y_{j-1}\right)\right]
\end{array}
$$

But it is difficult to ensure the accuracy if only the adjacent points will be compared.

3) The entropy comparison method. Where the entropy is 
defined as

$$
H_{f}=-\sum_{N=1}^{N} \sum_{M=1}^{M} f(x, y) \ln [f(x, y)]
$$

It is believed that image sharpness is proportional to the entropy. But the logarithmic calculation in the formula will take much time in DSP. This algorithm can't satisfy the real-time requirements in substantial amounts of monotonous code.

4) Sum of Laplace transforms. In this algorithm, we transform the image with the Laplace operator. After that, the sum of the result of processing across the whole image is regarded as the standard with which the image sharpness can be compared. This algorithm has a moderate computational complexity, and the accurancy is guaranteed.

After comparison of the above algorithm, we choose the sum of Laplace transforms as our core algorithm in the system. In addition to the reasons mentioned above, another main reason is that the core chip we use in this system is DSP, which multiply the most efficiency with the built-in multiplier, and the main computation in this algorithm is multiplication and additive. This algorithm can operate efficiently in this hardware.

\section{B. Climbing Algorithm}

Climbing algorithm, as one of the most commonly used control algorithm in automatic focusing, is chosen as the control algorithm. Climbing algorithm reduce the computation time by changing its stepping value in the process of adjusting focal length. It can achieve high efficiency in operation.

The theory of climbing algorithm can be illuminated as following (see Fig. 5).

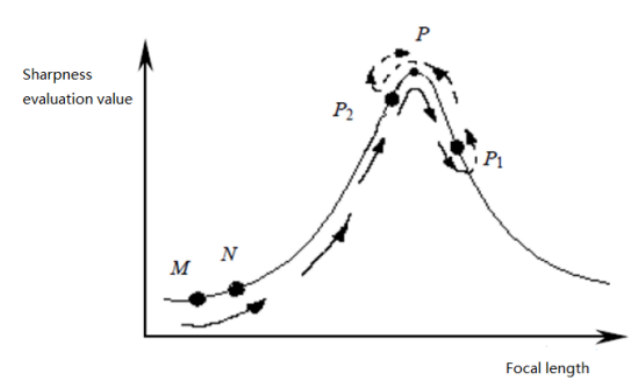

Fig. 5. The theory of climbing algorithm for autofocus.

The initial adjustment direction is determined by Point $\mathrm{M}$ and Point N. We adjust the focal length towards this direction with the largest stepping value until the sharpness evaluation value starts falling down. In the second step, we change the direction of adjustment with a smaller stepping value and stop when the sharpness evaluation value starts falling down again. Repeat the above work until we use the smallest stepping value and record the final position which is the most ideal focal length.

\section{EXPERIMENTS AND RESULTS}

From the analysis of climbing algorithm, it is obvious to see that there are two main influencing factors in the processing of climbing algorithm, one of them is the accuracy of sharpness evaluation value, the other one is the stability and accuracy of hardware. The paper is focus on the optimization of the two parameters, so we measured the two parameters in our experiment.

\section{A. Accuracy Evalution of Constant Current Source}

In the current test, the reference instrument was Thurlby-1905a. It's a digital multimeter with accuracy of $0.39 \mathrm{~mA}$ with the range of $200 \mathrm{~mA}$ and $3.9 \mathrm{~mA}$ within the range of $2 \mathrm{~A}$.

In order to evaluate the result intuitively, an USB driver of the Optotune lens was used to be a contrast test. We controlled both the two drivers to output a series of current, and measured their real output. Then the difference between the theoretical value and the actual value was recorded (see Fig. 6).

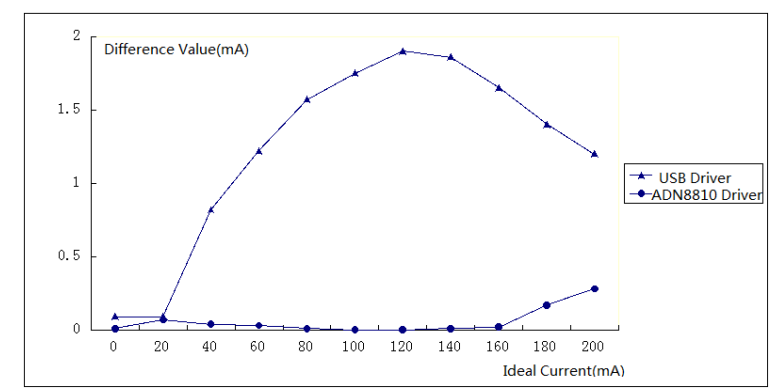

Fig. 6. The difference value of the two drivers.

As is shown in the figure, ADN8810 driver has an obvious improvement in the output precision. With a more precise driver, the system is able to control the focal length more accurately combined with the relationship between focal length of the liquid lens and real current.

What's more, the size of the driving hardware is $30.48 \times$ $18.42(\mathrm{~mm})$ which is smaller than the USB driver, so it can be more suitable for the system. This system can work well without the computer state with the hardware driver.

\section{B. Test for Sharpness Evaluation Function}

As the core of the system, sharpness evaluation function determines the accuracy of automatic focusing system. We tested the sum of Laplace transforms algorithm with a series of images with different sharpness which were collected by a zoom imaging system. We found the right focal length for the imaging system, and placed the images in the order of focal length, then evaluated the images with the algorithm.

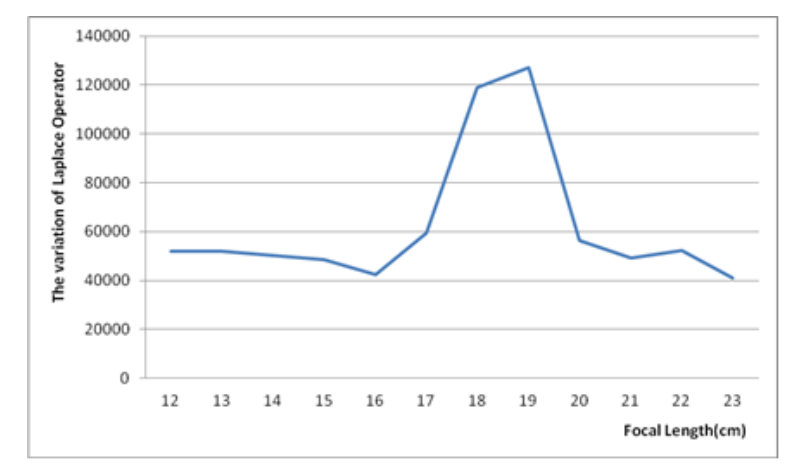

Fig. 7. The variation of Laplace Operator with the focus.

We recorded the result of the algorithm, and achieved the 
relationship between focal length and the evaluation results in Fig. 7.

This image fully meet the requirement of climbing algorithm, it has a peak when the image sharpness is the highest. We can finally find the right focal length with this algorithm accurately.

\section{CONCLUSIONS}

Thus, the entire system has been designed and tested. We can preliminarily prove the feasibility of this system. We can also improve automatic focusing process through the new hardware and algorithm.

The two factors which determine the system accuracy, sharpness evaluation value accuracy and hardware stability and accuracy, are both improved in this system. The confocal system can finally get a better result with the better control accuracy. Then the defocus problem that affects the sensitivity of the detecting system will be improved.

\section{ACKNOWLEDGMENT}

This work is supported by the Beijing Higher Education Young Elite Teacher Project (YETP1170).

\section{REFERENCES}

[1] E. Botcherby, C. Smith, M. Booth, R. Juskaitis, and T. Wilson, "Arbitrary-scan imaging for two-photon microscopy," in Proc. the Science and Industry Connect, 2010 , vol. 8.

[2] E. J. Botcherby, R. Juskaitis, M. J. Booth, and T. Wilson, "Aberration-free optical refocusing in high numerical aperture microscopy," Opt. Lett, vol. 32, no. 14, 2007.

[3] E. J. Botcherby, R. Juskaitis, M. J. Booth, and T. Wilson, "An optical technique for remote focusing in microscopy," Optics Community, vol. 281, no. 4, pp. 880-887, 2008.

[4] W. Göbel and F. Helmchen, "New angles on neuronal dendrites in vivo," J. Neurophysiol, vol. 98, no. 6, pp. 3770-3779, 2007.

[5] A. M. Kerlin, M. L. Andermann, V. K. Berezovskii, and R. C. Reid, "Broadly tuned response properties of diverse inhibitory neuron subtypes in mouse visual cortex," Neuron, vol. 67, no. 5, pp. 858-871, 2010.

[6] E. E. Hoover, M. D. Young, E. V. Chandler, A. Luo, J. J. Field, K. E. Sheetz, A. W. Sylvester, and J. A. Squier, "Remote focusing for programmable multi-layer differential multiphoton microscopy," Biomed. Opt. Express, vol. 2, no. 1, pp. 113-122, 2011.

[7] B. F. Grewe, F. F. Voigt, M. Hoff et al., "Fast two-layer two-photon imaging of neuronal cell populations using an electrically tunable lens," Biomedical Optics Express, 2011, vol. 2, no. 7, pp. 2035-2046.

[8] S. Casutt, M. Bueeler, M. Blum et al., "Fast and precise continuous focusing with focus tunable lenses," in Proc. the International Society for Optics and Photonics, 2014

[9] M. Blum, M. Büeler, C. Grätzel et al., "Optotune focus tunable lenses and laser speckle reduction based on electroactive polymers," in Proc. the International Society for Optics and Photonics, 2012.

[10] M. Blum, M. Büeler, C. Grätzel et al., "Compact optical design solutions using focus tunable lenses," in Proc. the Optical Systems Design. International Society for Optics and Photonics, 2011.

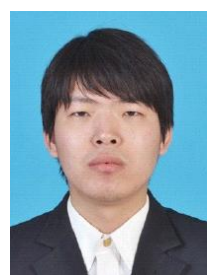

Chang Zhou received his BE degree in optoelectronics from Beijing Institute of Technology (BIT) in 2014. He has been studying for a master's degree in BIT since then.

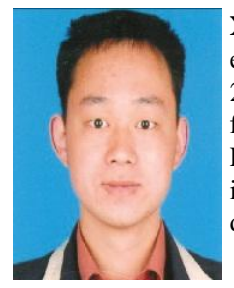

Xiaoming Hu received his BS degree in physical electronics from Beijing Institute of Technology (BIT) in 2001 and received his $\mathrm{PhD}$ degree in optical engineering from BIT in 2006. He has been working at the School of Life Science in BIT since then. His research interests include biomedical photonics, piezosurgery, and optical detection in microfluidics.

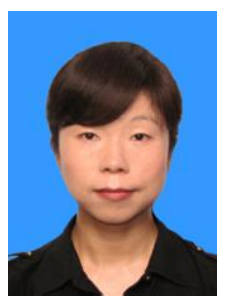

Ya Zhou received her Ph.D. degree in optical engineering from Beijing Institute of Technology, China, in 2000. After two years post-doctor research in Institute of Remote Sensing and Geography Information Systems, Peking University, she joined School of Optoelectronics of Beijing Institute of Technology as an associate professor in August 2002.

Dr. Zhou was working at Computer Department of North Carolina State University (NCSU, USA) as a visiting professor during September 2009 to March 